\title{
Initial experience, feasibility and safety of permanent left bundle branch pacing: results from a prospective single- centre study
}

\author{
L. M. Rademakers (D) - J. L. P. M. van den Broek · M. Op 't Hof · F. A. Bracke
}

Accepted: 23 September 2021 / Published online: 26 November 2021

(C) The Author(s) 2021

\begin{abstract}
Background Left bundle branch (LBB) pacing is a novel pacing technique which may serve as an alternative to both right ventricular pacing for symptomatic bradycardia and cardiac resynchronisation therapy (CRT). A substantial amount of data is reported by relatively few, highly experienced centres. This study describes the first experience of LBB pacing in a high-volume device centre.

Methods Success rates (i.e. the ability to achieve LBB pacing), electrophysiological parameters and complications at implant and up to 6 months of follow-up were prospectively assessed in 100 consecutive patients referred for various pacing indications.

Results The mean age was $71 \pm 11$ years and $65 \%$ were male. Primary pacing indication was atrioventricular (AV) block in $40 \%$, CRT in $42 \%$, and sinus node dysfunction or refractory atrial fibrillation prior to $\mathrm{AV}$ node ablation in 9\% each. Baseline left ventricular ejection fraction was $<50 \%$ in $57 \%$ of patients, mean baseline QRS duration $145 \pm 34 \mathrm{~ms}$. Overall LBB pacing was successful in 83 of 100 (83\%) patients but tended to be lower in patients with CRT pacing indication (69\%, $p=\mathrm{ns}$ ). Mean left ventricular activation time (LVAT) during LBB pacing was $81 \mathrm{~ms}$ and paced QRS duration was $120 \pm 19$ ms. LBB capture threshold and R-wave sense at implant was $0.74 \pm 0.4 \mathrm{mV}$ at $0.4 \mathrm{~ms}$ and $11.9 \pm 5.9 \mathrm{~V}$ and remained stable at 6-month follow-up. No complications occurred during implant or follow-up.

Conclusion LBB pacing for bradycardia pacing and resynchronisation therapy can be easily adopted by
\end{abstract}

L. M. Rademakers $(\bowtie)$ J. L. P. M. van den Broek ·

M. Op 't Hof · F. A. Bracke

Department of Cardiology, Catharina Hospital, Eindhoven, The Netherlands

nard.rademakers@catharinaziekenhuis.nl experienced implanters, with favourable success rates and safety profile.

Keywords Left bundle branch pacing - Physiologic pacing · Cardiac resynchronisation therapy · Bradycardia pacing

\section{Background}

For more than 60 years, permanent cardiac pacing for symptomatic bradycardia has been performed by endocardial stimulation of the right ventricle. Stimulating the right ventricle induces abnormal electrical activation and asynchronous ventricular contraction which may lead to adverse cardiac remodelling over time $[1,2]$. This has been associated with an increased risk of congestive heart failure (CHF), atrial fibrillation and cardiovascular mortality [3-8]. Pacing from the right ventricular septum and pacing from the right ventricular apex are equally prone to these complications [9].

Cardiac resynchronisation therapy (CRT) improves pump function, clinical status and reduces morbidity and mortality in patients with moderate-to-severe

\section{What's new?}

- Left bundle branch pacing is a novel pacing technique for bradycardia pacing and cardiac resynchronisation therapy.

- The majority of available data is reported by relatively few highly experienced centres.

- Left bundle branch pacing can be adopted quickly by operators without previous experience with high success and low complication rates. 


\section{Advertisement placed here.}

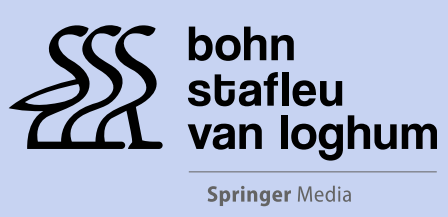

Houten 2021 


\section{Advertisement placed here.}

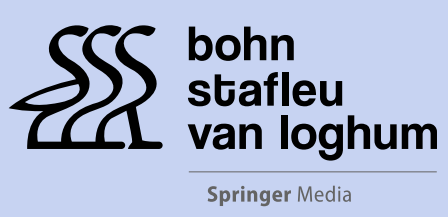

Houten 2021 
heart failure and left bundle branch block (LBBB) [10]. The left ventricular pacing lead is positioned via the coronary sinus at the epicardium of the left ventricle. Biventricular pacing creates an electrical activation pattern that is the composite of two wave fronts originating from the right and left ventricle. It offers only modest reduction in QRS duration and left ventricular activation time since activation of the ventricles utilises non-physiological, slow cell-to-cell conduction instead of the intrinsic His-Purkinje conduction system[11-13]. Non-response to treatment approximates $30 \%$ and may partly be due to suboptimal resynchronisation [10].

Physiologic pacing is characterised by direct stimulation of the intrinsic His-Purkinje system and results in physiologic ventricular depolarisation and repolarisation. In 2017, Huang et al. first demonstrated that, by pacing beyond the region of block, left bundle branch (LBB) pacing could achieve complete correction of LBBB and improved left ventricular function in a patient with heart failure and LBBB [14]. Since then, this technique has emerged as an alternative to both traditional right ventricular pacing for bradycardia and classic CRT [15-19]. Several publications demonstrated high success rates, although the majority of scientific literature has been reported by relatively few highly experienced operators and centres [15, 20-22]. This study describes the feasibility and the safety of permanent LBBP for various pacing indications in a high-volume referral centre with no previous experience with this new pacing technique.

\section{Methods}

\section{Patient selection}

The first 100 consecutive patients undergoing an attempt at LBB pacing at the Catharina Hospital between January 2020 and September 2020 were prospectively investigated. Indications for pacing included bradycardia (single-chamber or dual-chamber pacemaker) or CRT (CRT pacemaker [CRT-P] or defibrillator [CRT-D]). No pre-selection of patients based on pacing indication was made. Prior to the implantation procedure the operators discussed with the patients the nonstandard but potentially more physiological nature of conduction system pacing. All procedures followed our institutional guidelines and all patients provided informed consent. Two implanting physicians participated equally in this study (i.e. L.M.R. and F.A.B.).

\section{Procedure}

All device implantations were performed under local anaesthesia and after perioperative administration of $2 \mathrm{~g}$ of intravenous cefazolin. None of the patients underwent sedation or general anaesthesia. If patients were on direct oral anticoagulant therapy (DOAC), treatment was interrupted-as recommended-at least $24 \mathrm{~h}$ before implantation. Vitamin $\mathrm{K}$ antagonists were generally not interrupted, and device implantation was performed if the international normalised ratio (INR) did not exceed 3.0. Cephalic vein access for all leads using a modified Seldinger technique was the standard approach. Alternative access (i.e. axillary or subclavian vein puncture) was reserved as 'bailout' option.

\section{Left bundle branch lead implantation}

LBB pacing was performed using the SelectSecure Model 3830, $74 \mathrm{~cm}$ pacing lead (Medtronic Inc, Minneapolis, MN) and the C315HIS delivery sheath (Medtronic Inc, Minneapolis, MN). We based our technique on the descriptions by Huang [22]. After advancing the C315HIS sheath into the right ventricle, guided by an angled hydrophilic guidewire (Terumo Radifocus Guidewire M, 0.035", $120 \mathrm{~cm}$, Terumo Inc), the pacing lead was advanced through the sheath in right anterior oblique 20 degrees fluoroscopic projection. Unipolar pace mapping through the tip of the lead at $5 \mathrm{~V}$ was used to scan the right ventricular septum to find the optimal pacing site, i.e. (i) a paced ECG QRS morphology in lead V1 showing a "W" morphology with a mid-notch and/or (ii) the presence of inferior lead and aVR/aVL discordance ( $\mathrm{R}$ wave in lead II taller than in lead III, or a negative aVR and positive aVL). Fig. 1 schematically indicates finding the optimal pacing site. Using these criteria helped avoiding inadvertent lead fixation in the right ventricular outflow tract. In left anterior oblique 30 degrees fluoroscopic projection, the sheath was positioned perpendicular to the interventricular septum by anticlockwise rotation of the sheath and the pacing lead was fixated in the septum with 2 to 3 quick clockwise rotations while lead tip and sheath remained co-axial during lead fixation. Again, unipolar pacing was performed to confirm the presence of a good initial paced QRS morphology and to document the baseline pacing impedance. Subsequently, the lead was advanced by further clockwise rotations into the septum under intermittent fluoroscopic guidance. Unipolar pacing was performed after every few rotations to assess the paced QRS morphology and pacing impedance until the paced QRS morphology resembled a right bundle branch (RBB) block or RBB conduction delay pattern in V1 (QR pattern), or QS pattern with a narrow QRS. Lead depth into the interventricular myocardium was approximated by the position of the proximal radiopaque marker relative to the end of the C135HIS introducer sheath positioned against the interventricular septum. The distance between the tip of the helix and the proximal end of the radiopaque marker was approximately $1 \mathrm{~cm}$. Only clockwise rotations were used as anticlockwise rotations were regarded as a risk factor for lead dislodgment, i.e. the pacing lead not being fixated by the screw and just lying in a drilled 


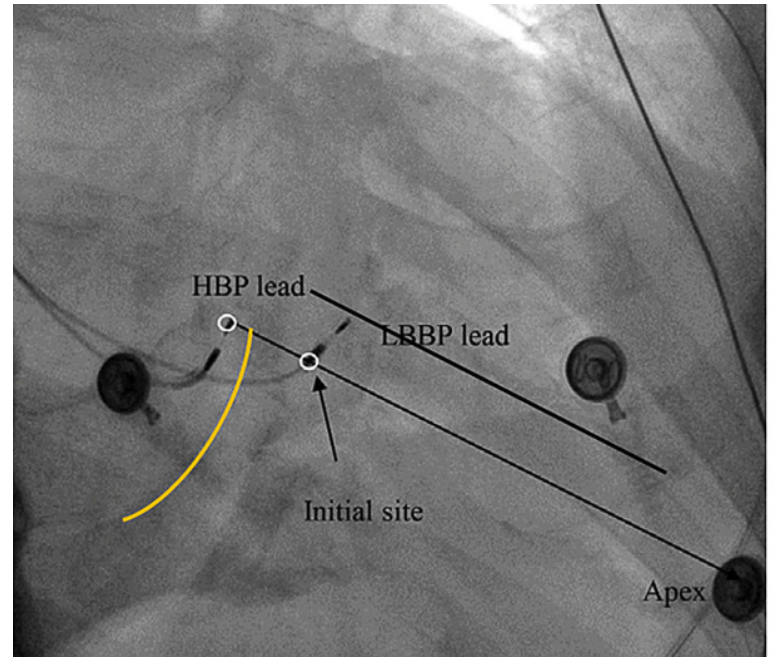

Fig. 1 How to locate the site for left bundle branch pacing (LBBP) and electrocardiogram characteristics. Left panel: location of the His-bundle pacing (HBP) lead and LBBP lead in the right anterior oblique 30 view. Yellow line indicates location of tricuspid valve and black lines indicate demarcation of

hole. We tested and recorded the left ventricular activation time (LVAT, measured as time interval from unipolar pacing spike to peak R wave at lead V5 or V6) at different outputs (usually at $1.5 \mathrm{~V}$ at $0.4 \mathrm{~ms}$ and at $5.0 \mathrm{~V}$ at $0.4 \mathrm{~ms}$ ) on an electrophysiology recording system (Prucka Cardiolab, GE Healthcare, Waukesha, Wisconsin). LBB pacing was confirmed when the paced QRS morphology demonstrated either an RBB block morphology (QR or rSR') or a QS pattern with narrow QRS $(<130 \mathrm{~ms})$, and LVAT that shortened abruptly with increasing output or remained shortest and constant both at low and high outputs. Although there is no validated cut-off yet, we regarded LVAT $\leq 90 \mathrm{~ms}$ as an indicator of LBB capture [23]. LBB pacing was regarded unsuccessful if the abovementioned criteria could not be met.

\section{Endpoints}

The endpoints of this study are the acute LBB pacing success rates and LBB pacing-related procedural complications within six months from implantation. The latter include interventricular septal perforation, septal coronary artery injury, transient ischaemic attack and/or stroke, lead dislodgment, device-related infection, clinically relevant decrease in R-wave sensing, or a $>50 \%$ decrease in R-wave sense, and an increase in LBB capture threshold $>2 \mathrm{~V}$.

\section{Data analysis}

Continuous data are presented as mean and standard deviation and discrete variables as counts and percentages, unless otherwise stated. Continuous data was compared using a Student's t-test. Discrete variables were analysed with chi-squared or Fisher's exact

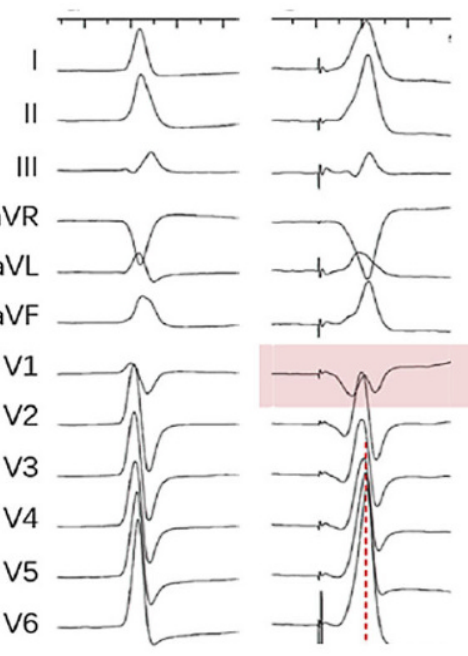

interventricular septum. Right panel: native conduction and unipolar pace mapping at the interventricular septum before lead fixation demonstrating morphology of "w" pattern with a notch at the nadir of the QRS in lead V1. Modified after Huang et al. [22] and Zhang et al. [25]

test. A 2 -sided $p$-value $<0.05$ was considered statistically significant. No missing data imputation was performed. Analyses were performed using SPSS Statistics (v.25, IBM Corp., Armonk, NY).

\section{Results}

Baseline characteristics are summarised in Tab. 1. The study population consisted of 100 consecutive patients who underwent an attempt at LBB pacing. Their mean age was 71 years and two-thirds were male. About $60 \%$ of patients had a left ventricular ejection fraction below 50\%. Pacing indications included sinus node dysfunction $(9 \%)$, atrioventricular (AV) block (40\%), refractory atrial fibrillation prior to AV node ablation (9\%), and CRT or right ventricular pacing induced heart failure $(42 \%)$. The mean baseline QRS duration was $146 \mathrm{~ms}$ and $43 \%$ of all patients had an LBBB (Tab. 1).

\section{Success rates and implantation characteristics}

In the 100 patients who had an attempt at LBB pacing, 83 were successful according to the predefined criteria. The success rate among patients referred for sinus node dysfunction, AV block or refractory atrial fibrillation prior to $\mathrm{AV}$ node ablation was $88 \%$ whereas in patients referred for CRT $(n=38)$ or pacing induced cardiomyopathy $(n=4)$ success rate was $69 \%(p=0.123)$. The success rates between patients with and without LBBB differed significantly ( $67 \%$ and $88 \%$ respectively, $p=0.047)$. The overall success rate was $78 \%$ in the first and $88 \%$ in the last 50 patients $(p=0.183)$. The mean procedural time (defined as door-to-door time) decreased in the latter 50 patients from $119 \pm 42 \mathrm{~min}$ to $104 \pm 33 \mathrm{~min}(p=0.111)$. There were no differences 
Table 1 Baseline characteristics

\begin{tabular}{|c|c|}
\hline \multicolumn{2}{|l|}{ Parameter } \\
\hline Age & $71 \pm 11$ \\
\hline \multicolumn{2}{|l|}{ Sex } \\
\hline Male & $67(67 \%)$ \\
\hline Female & $33(33 \%)$ \\
\hline \multicolumn{2}{|l|}{ Medical history } \\
\hline Hypertension & $41(41 \%)$ \\
\hline Chronic kidney disease & $6(6 \%)$ \\
\hline Diabetes mellitus & $18(18 \%)$ \\
\hline Coronary artery disease & $33(33 \%)$ \\
\hline Atrial fibrillation/flutter & $40(40 \%)$ \\
\hline LV dysfunction (EF < 50\%) & $60(60 \%)$ \\
\hline \multicolumn{2}{|l|}{ Pacing indication } \\
\hline Sinus node dysfunction & $9(9 \%)$ \\
\hline AV block & $40(40 \%)$ \\
\hline CRT or pacing-induced CMP & $42(42 \%)$ \\
\hline Refractory AF and/or AVN ablation & $9(9 \%)$ \\
\hline \multicolumn{2}{|l|}{ Electrocardiogram } \\
\hline QRS width, ms & $146 \pm 34$ \\
\hline Narrow QRS (<120ms) & $21(21 \%)$ \\
\hline Wide QRS ( $\geq 120 \mathrm{~ms}$ ) & 79 (79\%) \\
\hline - LBBB & $43(54 \%)$ \\
\hline - Escape rhythm, IVCD or RBBB & $21(27 \%)$ \\
\hline - RV pacing & $15(19 \%)$ \\
\hline \multicolumn{2}{|l|}{ Implanted device } \\
\hline Singe-chamber pacemaker & $6(6 \%)$ \\
\hline Dual-chamber pacemaker & $50(50 \%)$ \\
\hline Dual-chamber defibrillator & $3(3 \%)$ \\
\hline CRT-P & $9(9 \%)$ \\
\hline CRT-D & $32(32 \%)$ \\
\hline \multicolumn{2}{|c|}{$\begin{array}{l}\text { Values are } n,(\%) \text { or mean } \pm \text { standard deviation } \\
L V \text { left ventricular, } E F \text { ejection fraction, } A V \text { atrioventricular, } C R T \text { cardiac resyn- } \\
\text { chronisation therapy, } C M P \text { cardiomyopathy, } A F \text { atrial fibrillation, } A V N \text { atri- } \\
\text { oventricular node, } L B B B \text { left bundle branch block, } R B B B \text { right bundle branch } \\
\text { block, } I V C D \text { intraventricular conduction delay, } R V \text { right ventricular, } C R T-P \text { car- } \\
\text { diac resynchronisation therapy pacemaker, } C R T-D \text { cardiac resynchronisation } \\
\text { therapy defibrillator }\end{array}$} \\
\hline
\end{tabular}

in baseline characteristics or in pacing indication between the first and second half of the study group. The mean LVAT was $81 \mathrm{~ms}$ in the 83 successful attempts. There was no difference in LVAT between patients with or without LBBB $(83 \pm 15 \mathrm{~ms}$ and $80 \pm 13 \mathrm{~ms}$ respectively). In patients with previous right ventricular pacing there was a reduction of the mean paced QRS duration from $145 \pm 34 \mathrm{~ms}$ to $120 \pm 19 \mathrm{~ms}(p<0.001)$. In patients with baseline $\mathrm{LBBB}$, the mean reduction in QRS width after implantation was $42 \pm 19 \mathrm{~ms}$ (from $161 \pm 15 \mathrm{~ms}$ to $119 \pm 18 \mathrm{~ms}, p<0.001)$.

The total procedural duration was $103 \pm 30 \mathrm{~min}$ (median 101, interquartile range (IQR) 83-118) and the fluoroscopic duration for the entire procedure was $12 \pm 9$ min (median 9, IQR 6-16) (Tab. 2).

Tab. 3 presents follow-up data from device interrogation at 1 month and at 3 to 6 months. The mean follow-up time was $280 \pm 64$ days. The LBB
Table 2 Implantation data (data based on successful implants)

\begin{tabular}{l|l} 
Parameter & \\
LBB pacing attempted & 100 \\
\hline LBB pacing & \\
\hline - Successful & 83 \\
\hline - Not successful & 17 \\
\hline Total procedural duration, min & $103 \pm 30$ \\
\hline Fluoroscopy time, min & $12 \pm 9$ \\
\hline LV activation time & $81 \pm 14$ \\
\hline R-wave amplitude (mV) & $12 \pm 6$ \\
\hline Unipolar threshold at $0.4 \mathrm{~ms}(\mathrm{~V})$ & $0.7 \pm 0.4$ \\
\hline Impedance $(\Omega)$ & $739 \pm 154$ \\
\hline Baseline QRS duration & $145 \pm 34$ \\
\hline Paced QRS duration & $120 \pm 19$ \\
\hline Baseline QRS duration if LBBB & $161 \pm 15$ \\
\hline Paced QRS duration if LBBB & $119 \pm 18$ \\
\hline LBB left bundle branch, $L V$ left ventricular, LBBB left bundle branch block
\end{tabular}

capture threshold at implantation was $0.7 \pm 0.4 \mathrm{~V}$ and remained stable at 1 month and 3-6 months of follow-up $(0.7 \mathrm{~V} \pm 0.2 \mathrm{~V}$ and $0.8 \mathrm{~V} \pm 0.2$ respectively). The sensed $\mathrm{R}$ wave at implantation was $11.9 \pm 5.9 \mathrm{mV}$ and increased to $14.6 \pm 6.3 \mathrm{mV}$ and $13.8 \pm 5.4 \mathrm{mV}$ at 1 and 3-6 months respectively. Impedance at implantation decreased from $739 \Omega$ to $572 \Omega$ and $536 \Omega$ at 1 and 3-6 months respectively.

\section{Unsuccessful attempts}

Satisfactory LBB pacing could not be obtained in 17 patients (9 male). Of thew 17 patients, 11 were referred for CRT with baseline $\mathrm{LBBB}$, low ejection fraction $(35 \pm 11 \%)$ and enlarged left ventricular dimensions (left ventricular end-diastolic diameter $55 \pm 13 \mathrm{~mm})$. Six of these 11 patients had an anteroseptal myocardial infarction with local scarring on ultrasound ( $p=0.192)$.

The indication in the remaining 6 failed attempts consisted of AV block in 4 patients, sinus node dysfunction and refractory atrial fibrillation prior to $\mathrm{AV}$ node ablation in one each.

The main reasons for failed LBB pacing included (i) the inability to position the pacing lead deep enough into the interventricular septum, mostly due to fibrosis after anteroseptal myocardial infarction, (ii) inability to obtain LVAT $\leq 90 \mathrm{~ms}$ due to peripheral conduction block, (iii) inability to engage the septum at the desired septal location due to lack of stable contact of the delivery sheath at the interventricular septum and (iiiv) inadequate length of the sheath to reach the desired location on the septum. The latter two reasons were noticed especially in patients with enlarged cardiac chambers. In addition, with increasing dwelling time the amount of provided support of the delivery sheath seemed to decrease. 
Table 3 Follow-up device data

\begin{tabular}{|c|c|c|c|}
\hline Parameter & Baseline & 1 month & $3-6$ months \\
\hline R-wave amplitude (mV) & $11.9 \pm 5.9$ & $14.6 \pm 6.3$ & $13.8 \pm 5.4$ \\
\hline Threshold at $0.4 \mathrm{~ms}(\mathrm{~V})$ & $0.7 \pm 0.4$ & $0.7 \pm 0.2$ & $0.8 \pm 0.2$ \\
\hline Impedance $(\Omega)$ & $736 \pm 153$ & $572 \pm 82$ & $537 \pm 79$ \\
\hline
\end{tabular}

\section{Complications}

In the perioperative phase, there were no complications recorded, i.e. no cardiac tamponade, septal coronary artery injury, interventricular fistula or pocket haematomas.

During follow-up visits at 2 weeks, 1 month and at 3 to 6 months, no pocket infections, lead dislodgement or lead perforation occurred. In none of the patients a sudden increase in capture threshold $>1 \mathrm{~V}$ or loss of capture occurred. In addition, none of the patients presented with stroke/transient ischaemic attack. At the end of 6 months of follow-up there were no lead infections, lead dysfunctions or lead revisions and none of the leads had been extracted.

\section{Discussion}

The major findings of this prospective study in 100 consecutive patients are: (i) LBB pacing is feasible in $83 \%$ of a mixed population of indications and (ii) can safely be performed without complications with (iii) satisfactory electrical lead parameters which remain stable at medium-term follow-up.

The current study also shows that this novel pacing technique is easy to perform and quickly learned by experienced device implanters. In our opinion, the learning curve is quite steep and experienced operators should be able to reach a plateau phase within 30-50 LBB pacing attempts [20].

The technique of scanning the right ventricular septum by pace mapping while looking for a "W" morphology in the V1 lead in combination with inferior lead and aVR/aVL discordance [24] was easy to perform and facilitated localisation of the optimal site for LBB pacing and avoided inadvertent lead fixation in the right ventricular outflow tract.

LBB pacing could not be performed in 17 patients (17\%). Success rates were highest among patients with pacing indication for sinus node dysfunction, $\mathrm{AV}$ block or refractory atrial fibrillation prior to $\mathrm{AV}$ node ablation and lower in patients with CRT indication, an observation also reported by Padala et al. [24] Eleven of these patients had a CRT indication. Reasons for failure included patients with extensive septal myocardial scarring as extensive fibrosis prohibited advancement of the lead with the available implant tools. Furthermore, in some cases length and/or stability of the guiding sheath we used was insufficient and made it impossible to reach the desired location on the right ventricular septum in these dilated hearts. In patients with dilated cardiomyopathy, subclavian venous puncture may be preferable over cephalic venous cutdown since the distance from venous access to the heart is larger with the latter technique. Probably the use of a longer, steerable or firmer delivery sheath could overcome these shortfalls.

LBB capture was confirmed by only two markers, i.e. (i) the paced QRS morphology in lead V1 demonstrating RBB conduction delay or block pattern, or QS pattern with narrow QRS and (ii) a stable and short LVAT. The wide spectrum of QR, rSR', Qr, rS, and QS morphologies in lead V1 may be explained by various conduction patterns between transverse connections between the right and left bundles [25]. The LVAT was measured in precordial leads V5 or V6, whichever was longest, as a measure of the electrical activation time from the left ventricular endocardium to the epicardium of the left ventricular lateral wall. It should remain constant during both selective and non-selective LBB capture as a marker for LBB capture with fast activation propagation throughout the specialised conduction fascicules of the LBB. There are, however, no validated cut-off values of what the LVAT should be. In our cohort, the mean LVAT was $81 \mathrm{~ms}$ and is similar to previously reported data $[16,26,27]$. However, in patients with diffuse peripheral conduction disease, LVAT values may be prolonged, even with LBB capture. LVAT may also be extended in patients with dilated cardiomyopathy where path length to the left ventricular lateral wall is increased.

We did not include the presence of an LBB potential as criterion for LBB capture. Previous studies reported a high variability in recording these potentials, from $28 \%$ to $80 \%$ of cases $[21,28,29]$. In patients with LBBB, LBB potentials can only be recorded during restoration of left bundle conduction, e.g. by temporary Hisbundle pacing [27]. In addition, Padala et al. demonstrated in a recent series of 305 cases that an LBB potential could be found in only $41 \%$ of patients with successful LBB lead implantation [24]. In their study, only $11 \%$ of patients had baseline LBBB. In our study just over $40 \%$ of patients had an LBBB. It may be questionable whether documenting an LBB potential is a prerequisite of confirmation of LBB capture [28, 30]. At the optimal pacing sites, LBB capture threshold is almost invariably lower than local myocardial capture and could be helpful in differentiating selective versus non-selective LBB pacing. The resulting difference in QRS morphology during unipolar pacing is also an indication for LBB capture as changing pacing output would not change the QRS morphology with myocardial only stimulation. Since we did not record LBB potentials, determination of selective 
or non-selective LBB pacing (i.e. direct activation of both LBB and local myocardium) was not possible. However, Su et al. recently published that in 460 patients with selective LBB capture at implant, at followup 191 patients still had selective LBB capture whereas 292 patients had non-selective LBB capture, which is probably caused by a decrease in septal myocardial capture threshold in the post-operative phase [20].

The short-term pacing and sensing parameters were excellent and compared favourable to traditional right ventricular pacing. During follow-up, the lead parameters remained very stable. Probably lead maturation may benefit from a deep intramyocardial lead position when compared to traditional endocardial leads.

In this study neither implantation related nor lead performance or device related complications were recorded during the entire follow-up. Importantly, results should be interpreted with caution since sample size was relatively small and follow-up short.

\section{Unknowns and future perspectives}

Mechanical effects of the contracting myocardium on the deep-seated lead body may affect lead longevity and needs to be further studied. In addition, the ability to safely extract these leads in the future should be investigated [14].

Although LBB pacing seems a potential alternative to conventional CRT in patients with LBBB, it is still unknown which patients will benefit most from this novel technique. Upadhyay et al. showed that LBBB with intact Purkinje activation was present in approximately only $36 \%$ patients in their study [31]. The presence of conduction delay distally from the pacing site may attenuate effective resynchronisation. However, regions of conduction delay or block may also influence the effectiveness of coronary sinus left ventricular pacing. In contrast, if the LBBB can be corrected by LBB pacing, the region of block is most likely situated proximally of the pacing site and with intact distal Purkinje conduction [31]. Indeed, in patients with successful LBB pacing mean LVAT values were similar when comparing baseline LBBB and non-LBBB (83 ms versus $81 \mathrm{~ms}$ respectively).

The predictive value of demonstrating scar tissue in the septal region of the LBB on successful LBB pacing should also be investigated as this could prevent futile attempts at LBB pacing, especially in patients with ischaemic cardiomyopathy.

The current delivery sheath and lead were not specifically developed for LBB pacing and new developments in delivery sheath and possibly lead design may improve the success rate. In addition, the use of stylet-driven leads may further increase the success rate of LBB pacing. Concerns about the use of these leads include their larger diameter in comparison to the SelectSecure Model 3830 lead used in this study. Larger leads may carry additional risk of perforation of septal coronary artery branches. In addition, extendable screws lack the isodiametric shape at the location where the helix exits the helix case, which might limit lead penetration into the septum. Longterm data on lead behaviour in deep septal position and lead performance are still lacking for stylet-driven leads.

Finally, large prospective randomised studies are needed to further confirm the feasibility, long-term safety and usefulness of LBB pacing for various conditions.

\section{Conclusions}

This prospective single-centre study demonstrated that LBB pacing for bradycardia pacing and resynchronisation therapy can be adopted quickly by a centre without previous experience with this technique with favourable success rates and safety profile.

Funding No funding was received for this study.

Conflict of interest L.M. Rademakers, J.L.P.M. van den Broek, M. Op 't Hof and F.A. Bracke declare that they have no competing interests.

Open Access This article is licensed under a Creative Commons Attribution 4.0 International License, which permits use, sharing, adaptation, distribution and reproduction in any medium or format, as long as you give appropriate credit to the original author(s) and the source, provide a link to the Creative Commons licence, and indicate if changes were made. The images or other third party material in this article are included in the article's Creative Commons licence, unless indicated otherwise in a credit line to the material. If material is not included in the article's Creative Commons licence and your intended use is not permitted by statutory regulation or exceeds the permitted use, you will need to obtain permission directly from the copyright holder. To view a copy of this licence, visit http://creativecommons.org/licenses/by/4.0/.

\section{References}

1. Vassallo JA, Cassidy DM, Miller JM, et al. Left ventricular endocardial activation during right ventricular pacing: effect of underlying heart disease. J Am Coll Cardiol. 1986;7:1228-33.

2. Prinzen FW, Peschar M. Relation between the pacing induced sequence of activation and left ventricular pump function in animals. Pacing Clin Electrophysiol. 2002;25:484-98.

3. Leong DP, Mitchell AM, Salna I, et al. Long-term mechanical consequences of permanentright ventricular pacing: effect of pacing site. J Cardiovasc Electrophysiol. 2010;21:1120-6.

4. Shibayama J, Yuzyuk TN, Cox J, et al. Metabolic remodeling in moderate synchronous versus dyssynchronous pacinginduced heart failure: integrated metabolomics and proteomics study. PLoS ONE. 2015;10:e118974.

5. Nielsen JC, Kristensen L, Andersen HR, et al. A randomized comparison of atrial and dual-chamber pacing in 177 consecutive patients with sick sinus syndrome: echocardiographic and clinical outcome. J Am Coll Cardiol. 2003;42:614-23. 
6. Sweeney MO, Hellkamp AS, Ellenbogen KA, et al. Adverse effect of ventricular pacing on heart failure and atrial fibrillation among patients with normal baseline QRS duration in a clinical trial of pacemaker therapy for sinus node dysfunction. Circulation. 2003;107:2932-7.

7. Wilkoff BL, Cook JR, Epstein AE, et al. Dual-chamber pacing or ventricular backup pacing in patients with an implantable defibrillator: the Dual Chamber and VVI Implantable Defibrillator (DAVID) Trial. JAMA. 2002;288:3115-23.

8. Khurshid S, Epstein AE, Verdino RJ, et al. Incidence and predictors of right ventricular pacing-induced cardiomyopathy. Heart Rhythm. 2014;11:1619-25.

9. Spath NB, Wang K, Venkatasumbramanian S, et al. Complications and prognosis of patients undergoing apical or septal right ventricular pacing. Open Heart. 2019;6:e962.

10. Abraham WT, Fisher WG, Smith AL, et al. Cardiac resynchronization in chronic heart failure. $\mathrm{N}$ Engl J Med. 2002;346:1845-53.

11. Gervais R, Leclercq C, Shankar A, et al. Surface electrocardiogram to predict outcome in candidates for cardiac resynchronization therapy: a sub-analysis of the CARE-HF trial. Eur J Heart Fail. 2009;11:699-705.

12. Ploux S, Eschalier R, Whinnett ZI, et al. Electrical dyssynchrony induced by biventricular pacing: implications for patientselection and therapyimprovement. Heart Rhythm. 2015;12:782-91.

13. Jones S, Lumens J, Sohaib SMA, et al. Cardiac resynchronization therapy: mechanisms of action and scope for further improvement in cardiac function. Europace. 2017;19:1178-86.

14. Huang W, Su L, Wu S, et al. A novel pacing strategy with low and stable output: pacing the left bundle branch immediately beyond the conduction block. Can J Cardiol. 2017;33:1736 el-1736 e3.

15. Li X, Li H, Ma W, et al. Permanent left bundle branch area pacing for atrioventricular block: feasibility, safety, and acute effect. Heart Rhythm. 2019;16:1766-73.

16. Li Y, Chen K, Dai Y, et al. Left bundle branch pacing for symptomatic bradycardia: implant success rate, safety, and pacing characteristics. Heart Rhythm. 2019;16:1758-65.

17. Zhang W, Huang J, Qi Y, et al. Cardiac resynchronization therapy by left bundle branch area pacing in patients with heart failure and left bundle branch block. Heart Rhythm. 2019;16:1783-90.

18. Wu S, Su L, Vijayaraman P, et al. Left bundle branch pacing for cardiac resynchronization therapy: nonrandom- ized on-treatment comparison with his bundle pacing and biventricular pacing. Can J Cardiol. 2021;37:319-28.

19. Wang Y, Gu K, Qian Z, et al. The efficacy of left bundle branch area pacing compared with biventricular pacing in patients with heart failure: A matched case-control study. J Cardiovasc Electrophysiol. 2020;31:2068-77.

20. Su L, Wang S, Wu S, et al. Long-term safety and feasibility of left bundle branch pacing in a large single center study. Circ Arrhythm Electrophysiol. 2021;14:e9261.

21. Vijayaraman P, Subzposh FA, Naperkowski A, et al. Prospective evaluation of feasibility and electrophysiologic and echocardiographic characteristics of left bundle branch area pacing. Heart Rhythm. 2019;16:1774-82.

22. Huang W, Chen X, Su L, et al. A beginner's guide to permanent left bundle branch pacing. Heart Rhythm. 2019;16:1791-6.

23. Vijayaraman P, Ponnusamy S, Cano O, et al. Left bundle branch area pacing for cardiac resynchronization therapy: results from the international LBBAP collaborative study group. JAm Coll CardiolClin Electrophysiol. 2021;7:135-47.

24. Padala SK, Master VM, Terricabras M, et al. Initial experience, safety, and feasibility of left bundle branch area pacing: a multicenter prospective study. J Am Coll Cardiol Clin Electrophysiol. 2020;6:1773-82.

25. Zhang S, ZhouX, Gold MR. Left bundle branch pacing: JACC reviewtopic of the week. JAm CollCardiol. 2019;74:3039-49.

26. Chen K, Li Y. How to implant left bundle branch pacing lead in routine clinical practice. J Cardiovasc Electrophysiol. 2019;30:2569-77.

27. Chen X, Wu S, Su L, Su Y, Huang W. The characteristics of the electrocardiogram and the intracardiac electrogram in left bundle branch pacing. J Cardiovasc Electrophysiol. 2019;30:1096-101.

28. Guo J, Li L, Meng F, et al. Short-term and intermediateterm performance and safety of left bundle branch pacing. JCardiovasc Electrophysiol. 2020;31:1472-81.

29. Jiang Z, Chang Q, Wu Y, et al. Typical BBB morphology and implantation depth of 3830 electrode predict QRS correction by left bundle branch area pacing. Pacing Clin Electrophysiol. 2020;43:110-7.

30. Jastrzebski M, Moskal P, Bednarek A, et al. Programmed deep septal stimulation: a novel maneuver for the diagnosis of left bundle branch capture during permanent pacing. J Cardiovasc Electrophysiol. 2020;31:485-93.

31. Upadhyay GA, Cherian T, Shatz DY, et al. Intracardiac Delineation of Septal Conduction in Left Bundle-Branch Block Patterns. Circulation. 2019;139:1876-88. 